\title{
Representation of the auroral and polar ionosphere in the International Reference Ionosphere (IRI)
}

This issue of Advances in Space Research presents a selection of papers that document the progress in developing and improving the International Reference Ionosphere (IRI), a widely used standard for the parameters that describe the Earth's ionosphere. The core set of papers was presented during the 2010 General Assembly of the Committee on Space Research in Bremen, Germany in a session that focused on the representation of the auroral and polar ionosphere in the IRI model. In addition, papers were solicited and submitted from the scientific community in a general call for appropriate papers.

The auroral and polar region of Earth is where precipitating particles and open magnetic field lines cause a multitude of effects in the ionosphere that are not all yet fully understood and classified. An accurate representation of this highly dynamical region is a major challenge for a data-based model like the IRI. The papers published here include a first step towards this goal. Additionally, several papers investigate the performance of the IRI model during the recent highly unusual solar minimum that reached very low values in solar EUV irradiance and lasted almost double as long as prior minima. This issue continues a series of issues of Advances in Space Research dedicated to the International Reference Ionosphere, a joint project of the Committee on Space Research (COSPAR) and the International Union of Radio Science (URSI). A listing of prior issues and more information about the IRI project as well as access to the IRI code and interface can be found on the IRI homepage at http://iri.gsfc.nasa.gov/.
We thank the authors for their diligent work in preparing and revising their contributions. Each manuscript was evaluated by two reviewers. Not enough can be said about the important contribution made by our reviewing colleagues! They have spent their free time to help improve the quality of this issue. For this service we offer our heartfelt thanks. A list of those reviewers who agreed to be identified is at the end of this special issue.

Guest Editors

Dieter Bilitza

School of Physics Astronomy and Computational Science, George Mason University, Fairfax, VA 22020, USA Heliospheric Science Laboratory, Code 672, Goddard Space Flight Center, Greenbelt, MD 20771, USA E-mail address: dieter.bilitza-1@nasa.gov

Bodo Reinisch Center for Atmospheric Research, University of Massachusetts,

Lowell, MA 01854, USA Lowell Digisonde International, LLC, Lowell, MA 01854, USA E-mail address: bodo.reinisch@digisonde.com Available online 12 December 2012 